\title{
Properties of copper (fluoro-)phthalocyanine layers deposited on epitaxial graphene
}

\section{Citation}

Ren, Jun, Sheng Meng, Yi-Lin Wang, Xu-Cun Ma, Qi-Kun Xue, and Efthimios Kaxiras. 2011. "Properties of Copper (Fluoro-)Phthalocyanine Layers Deposited on Epitaxial Graphene." The Journal of Chemical Physics 134 (19): 194706. https://doi.org/10.1063/1.3590277.

\section{Permanent link}

http://nrs.harvard.edu/urn-3:HUL.InstRepos:41384024

\section{Terms of Use}

This article was downloaded from Harvard University's DASH repository, and is made available under the terms and conditions applicable to Other Posted Material, as set forth at http:// nrs.harvard.edu/urn-3:HUL.InstRepos:dash.current.terms-of-use\#LAA

\section{Share Your Story}

The Harvard community has made this article openly available.

Please share how this access benefits you. Submit a story.

Accessibility 


\title{
Properties of copper (fluoro-)phthalocyanine layers deposited on epitaxial graphene
}

\author{
Jun Ren, ${ }^{1}$ Sheng Meng, ${ }^{1,2, a)}$ Yi-Lin Wang, ${ }^{2}$ Xu-Cun Ma, ${ }^{2}$ Qi-Kun Xue, ${ }^{2,3}$ \\ and Efthimios Kaxiras ${ }^{1,4}$ \\ ${ }^{1}$ Insitut des Matériaux, École Polytechnique Fédérale de Lausanne (EPFL), CH-1015 Lausanne, Switzerland \\ ${ }^{2}$ Beijing National Laboratory for Condensed Matter Physics and Institute of Physics, Chinese Academy of \\ Sciences, Beijing 100190, China \\ ${ }^{3}$ Department of Physics, Tsinghua University, Beijing 100084, China \\ ${ }^{4}$ Department of Physics and School of Engineering and Applied Sciences, Harvard University, Cambridge, \\ Massachusetts 02138, USA
}

(Received 19 February 2011; accepted 22 April 2011; published online 19 May 2011)

\begin{abstract}
We investigate the atomic structure and electronic properties of monolayers of copper phthalocyanines $(\mathrm{CuPc})$ deposited on epitaxial graphene substrate. We focus in particular on hexadecafluorophthalocyanine $\left(\mathrm{F}_{16} \mathrm{CuPc}\right)$, using both theoretical and experimental (scanning tunneling microscopy $\mathrm{STM}$ ) studies. For the individual $\mathrm{CuPc}$ and $\mathrm{F}_{16} \mathrm{CuPc}$ molecules, we calculated the electronic and optical properties using density functional theory (DFT) and time-dependent DFT and found a red-shift in the absorption peaks of $\mathrm{F}_{16} \mathrm{CuPc}$ relative to those of $\mathrm{CuPc}$. In $\mathrm{F}_{16} \mathrm{CuPc}$, the electronic wavefunctions are more polarized toward the electronegative fluorine atoms and away from the $\mathrm{Cu}$ atom at the center of the molecule. When adsorbed on graphene, the molecules lie flat and form closely packed patterns: $\mathrm{F}_{16} \mathrm{CuPc}$ forms a hexagonal pattern with two well-ordered alternating $\alpha$ and $\beta$ stripes while $\mathrm{CuPc}$ arranges into a square lattice. The competition between molecule-substrate and intermolecular van der Waals interactions plays a crucial role in establishing the molecular patterns leading to tunable electron transfer from graphene to the molecules. This transfer is controlled by the layer thickness of, or the applied voltage on, epitaxial graphene resulting in selective $\mathrm{F}_{16} \mathrm{CuPc}$ adsorption, as observed in STM experiments. In addition, phthalocyanine adsorption modifies the electronic structure of the underlying graphene substrate introducing intensity smoothing in the range of 2-3 eV below the Dirac point $\left(E_{D}\right)$ and a small peak in the density of states at $\sim 0.4 \mathrm{eV}$ above $E_{D}$. (C) 2011 American Institute of Physics. [doi:10.1063/1.3590277]
\end{abstract}

\section{INTRODUCTION}

The interaction between organic molecules and solid surfaces plays a central role in many technologically important applications such as molecular electronics, organic solar cells, and biosensors. For instance, organic solar cells are based on organic molecules and their interfaces with solid electrodes and have attracted growing attention due to their potential applications in low cost, environment friendly and flexible large-scale photovoltaic devices. Their energy conversion efficiency depends sensitively on the interface structure and electronic coupling between molecules and the electrode surface and between organic layers, and has increased significantly over the past decades due to the invention of donor-acceptor heterojunctions. ${ }^{1-4}$ Much current research has focused on understanding and controlling the interactions at the organic/inorganic interface, ${ }^{5-8}$ with a great deal of effort devoted to growing high-quality organic thin films by manipulating molecular orientation on solid substrates in order to enhance light absorption, control the type and concentration of interface carriers, and improve electron transfer at the interfaces. ${ }^{9-13}$

\footnotetext{
a) Author to whom correspondence should be addressed. Electronic mail: smeng@iphy.ac.cn.
}

Phthalocyanines (Pc's) and their derivatives, a class of aromatic compounds and a major component in various types of organic solar cells, have received much attention over the past decade. Pc molecules not only absorb in the red region of the solar spectrum, but are also highly stable organic semiconductors, which makes them suitable for energy conversion in organic solar cells. Copper phthalocyanine $(\mathrm{CuPc})$ is often used as an electron-donor material in contact with materials that have high electron affinity such as the fullerene $\mathrm{C}_{60} .{ }^{14,15}$ A sizable charge transfer occurs from metal substrates such as $\mathrm{Al}$, to $\mathrm{CuPc}$ at the metal-organic interface, ${ }^{16,17}$ while little charge transfer was observed at the interface between $\mathrm{CuPc}$ and highly oriented pyrolytic graphite (HOPG). ${ }^{18,19}$ In contrast, a thin film of copper hexadecafluorophthalocyanine, $\mathrm{F}_{16} \mathrm{CuPc}$, is a promising $n$-type $\pi$-conjugated organic semiconductor ${ }^{15,20}$ employed as an electron acceptor. Novel devices based on a $\mathrm{CuPc} / \mathrm{F}_{16} \mathrm{CuPc} p-n$ heterojunction have been fabricated for photovoltaic applications. ${ }^{21}$

Graphene, an atomically flat two-dimensional singlelayer of $\mathrm{C}$ atoms arranged in a honeycomb lattice, has emerged as a promising material for next generation electronic devices due to its interesting physical properties. ${ }^{22-24}$ With the rapid development of graphene technology in the past few years, high-quality large-scale graphene films can be produced and the carrier type and concentration in graphene 
can be precisely controlled. ${ }^{23}$ Therefore, it is highly promising to use graphene and its derivatives in organic solar cells as, for example, a nanoscale electrode, ${ }^{25}$ to assist donor/acceptor molecular assembly and carrier transfer. In addition, as a single atomic layer of carbon atoms, graphene is the simplest model to explore the interactions between layers of organic molecules and the electrode surface in thin-film based devices.

The interaction between Pc molecules and inorganic surfaces, including the single-crystal metal surfaces $\mathrm{Cu}(111)$, $\mathrm{Au}(111)$, and $\operatorname{Ag}(111),{ }^{12,26-29} \mathrm{HOPG}^{30,31}$ and the insulating oxide surface $\mathrm{SiO}_{2}$ (Refs. 32 and 33) has been intensively investigated. Most of the previous works focused primarily on experimental studies of the interface structure and molecular orientations. Recently, Wang et al. ${ }^{34}$ have employed scanning tunneling microscopy (STM) techniques to study the adsorption of $\mathrm{F}_{16} \mathrm{CuPc}$ molecules on monolayer and bilayer epitaxial graphene (EG) grown on a SiC substrate and observed well-ordered phases of incommensurate Pc islands. Here we investigate the structure of a Pc layer on EG at the atomic level and the nature of the interaction between the Pc molecules and EG, which are important in order to understand the charge transfer mechanisms at the organic/inorganic interface in molecular electronic devices and solar cells.

We use first-principles calculations based on density functional theory (DFT) and time-dependent DFT (TDDFT) to compare the electronic and optical properties of isolated $\mathrm{CuPc}$ and $\mathrm{F}_{16} \mathrm{CuPc}$ molecules. We then investigate possible configurations of $\mathrm{CuPc}$ and $\mathrm{F}_{16} \mathrm{CuPc}$ molecule on the graphene surface. After optimizing the structures, we found that Pc molecules are physisorbed on EG, lying flat to form well-ordered hexagonal $\left(\mathrm{F}_{16} \mathrm{CuPc}\right)$ or square lattices $(\mathrm{CuPc})$. A small amount of charge transfer from the EG to the Pc layer occurs upon adsorption; more importantly, the amount of charge transfer can be controllably tuned by the thickness of EG layers or the applied voltage on graphene, which results in the observed selective adsorption on monolayer graphene. $\mathrm{F}_{16} \mathrm{CuPc}$ exhibits a stronger polarization effect at the Pc/graphene interface than $\mathrm{CuPc}$. Local electronic features in the range of $2-3 \mathrm{eV}$ below and $0.4 \mathrm{eV}$ above the Dirac point $\left(E_{D}\right)$ are identified and compared to experiment, revealing the modification of the electronic structure of graphene due to Pc adsorption. The simulated STM images, optical spectra and molecular orbital alignments agree well with available experimental results. All these findings point to the possibility of controlling the adsorption structure and the amount of charge transfer from the substrate to the molecular layer at the organic/graphene interface which constitutes a novel approach to interfacial structure engineering and carrier control, in essence achieving controlled doping without chemical substitution.

\section{METHODS}

The first-principles calculations were performed with the SIESTA code ${ }^{35}$ and the Vienna $a b$ initio simulation package (VASP), ${ }^{36}$ in the framework of DFT. The supercell with periodic boundary conditions is composed of graphene and the monolayer of Pc molecules, with a C-C bond length of
$1.42 \AA$ and a vacuum layer exceeding $14 \AA$. For CuPc, we adopted an almost square lattice, with a graphene supercell $(1,5) \times(4,3)$, containing 78 carbon atoms per unit cell (UC). Here, $(n, m)$ denotes a vector which is a sum of $n$ and $m$ multiples of the two primitive lattice vectors of the graphene lattice. The almost square lattice vectors are $13.69 \AA$ and $14.96 \AA$, respectively, at an angle of $86^{\circ}$. This is the closest to a square cell that can be constructed as a supercell of the graphene lattice to match experimental observations of $\mathrm{Pc}$ on graphite. ${ }^{37}$ For the uniform $\mathrm{F}_{16} \mathrm{CuPc}$ layer, we adopted two almost hexagonal lattices to simulate two distinct arrangements of the molecules with respect to the graphene substrate: (1) The first has the Pc-Pc axis along the armchair direction of graphene and $(3,4) \times(4,3)$ lattice vectors which have a length of $14.96 \AA$ at an angle of $69^{\circ}$; this unit cell contains 80 carbon atoms per UC and is consistent with experimental observations $^{34}$ in this structure. (2) The second has the Pc-Pc axis along the zigzag direction of graphene, and lattice vectors $(6,0) \times(6,1)$, which are $14.76 \AA$ and $16.13 \AA$, at an angle of $68^{\circ}$; this unit cell includes 84 carbon atoms and is used for comparison. For the non-uniform $\mathrm{F}_{16} \mathrm{CuPc}$ layer, a unit cell with lattice vectors $(3,4) \times(8,6)$ is used to simulate the alternating $\alpha$ and $\beta$ stripes, containing two $\mathrm{F}_{16} \mathrm{CuPc}$ molecules per UC.

In the SIESTA calculations, we use pseudopotentials of the Troullier-Martins type ${ }^{38}$ to model the atomic cores, the Ceperley-Alder form of the local density approximation (LDA) as the exchange-correlation functional, ${ }^{39}$ and a local basis set of double- $\zeta$ polarized orbitals (13 orbitals for $\mathrm{C}, \mathrm{N}$, $\mathrm{F}$, and $\mathrm{O}$ and 5 orbitals for $\mathrm{H}$ ). Since van der Waals (vdW) forces are important for such weakly interacting systems, we also use vdW-density functionals (vdW-DF) of the LunqvistLangreth type ${ }^{40}$ for typical binding configurations. VdW-DF has been implemented in the SIESTA (Ref. 41) code by factorizing the integration kernel and using fast Fourier transforms to evaluate the self-consistent potential in $O(N \log (N))$ operation steps. Although there are other choices of functionals for dealing with dispersion interactions, the vdW-DF formulation of such interactions has proven successful in dealing with layered or sparse structures and more general geometries in which van der Waals interactions dominate; in particular it corrects binding energies which are usually underestimated in local/semi-local functionals. ${ }^{42}$ Moreover, the computation of dispersion interactions using this functional has reasonable computational cost, making it a practical option for studying relatively large systems as is the case in the present work. Although LDA systematically underestimates the binding energy for van der Waals dominated systems, it provides qualitatively correct energy differences and trends for interactions. An auxiliary real space grid equivalent to a plane-wave cutoff of $120 \mathrm{Ry}$ is employed in the calculation and spin polarization is taken into account. The $\Gamma$ point is used to optimize the structure, and a k-point mesh of $(8 \times 8 \times 1)$ in the Monkhorst-Pack sampling scheme is used to analyze the relevant electronic properties for the unit cell of the Pc/graphene system. For geometry optimization, the atomic structure is considered fully relaxed when the magnitude of forces on the atoms is smaller than $0.04 \mathrm{eV} / \AA$. The basis-set superposition error is excluded in the adsorption 
energies. The optical absorbance spectra is calculated from TDDFT in the linear response regime, ${ }^{43}$ by propagating the wave functions for 6107 steps in time with a time step of $3.4 \times 10^{-3} \mathrm{fs}$, which gives an energy resolution of $0.1 \mathrm{eV}$. The perturbing external field is 0.1 V/Å. The Heyd-ScuseriaErnzerhof (HSE) hybrid functional ${ }^{4,45}$ implemented in the VASP code was also used for the calculation of electronic states in the isolated molecules. In these VASP calculations, we used a plane-wave energy cut-off of $400 \mathrm{eV}$, a single kpoint $(\Gamma)$ for reciprocal-space sampling since the molecules are well isolated from their periodic images in a large unit cell of size $(20 \times 20 \times 10) \AA^{3}$, and the projector-augmented wave (PAW) pseudopotentials ${ }^{46}$ to represent the atomic cores. The atomic geometry obtained from the VASP calculation with the HSE functional is very similar to that obtained from the SIESTA calculation with the LDA functional. For instance, the average $\mathrm{Cu}-\mathrm{N}(\mathrm{C}-\mathrm{F})$ bond length in $\mathrm{F}_{16} \mathrm{CuPc}$ is $1.967 \AA$ $(1.330 \AA)$ in the VASP calculation and $1.944 \AA$ (1.328 $\AA)$ in the SIESTA calculation. A detailed comparison of atomic geometries as obtained by the HSE and LDA functionals is beyond the scope of the present work; such a comparison for similar systems can be found in Ref. 47.

The experiments were performed in a Unisoku ultrahigh vacuum low-temperature scanning tunneling microscopy system, which is equipped with a molecular beam epitaxy chamber for in situ sample preparation. EG was prepared on nitrogen doped $6 \mathrm{H}-\mathrm{SiC}(0001)$ substrate using solid state graphitization. Coexisting monolayer EG (MEG) and bilayer EG (BEG) surfaces were formed at the graphitization temperature $\mathrm{T}=1600 \mathrm{~K}$, on which $\mathrm{F}_{16} \mathrm{CuPc}$ molecules were deposited. $\mathrm{F}_{16} \mathrm{CuPc}$ molecules (Sigma-Aldrich) were evaporated from a Knudsen cell onto EG at room temperature. The deposition flux rate was $0.01 \mathrm{ML} / \mathrm{min}$. Here, 1 $\mathrm{ML}$ is defined as the surface coverage of the most closely packed $\mathrm{F}_{16} \mathrm{CuPc}$ monolayer. From the STM images discussed below, we can estimate that the molecular coverage in the closely packed $\mathrm{F}_{16} \mathrm{CuPc}$ islands is $\left(10^{8} \AA\right)^{2} / \mathrm{cm}^{2} /(15 \AA$ $\left.\times 16 \AA \times \sin \left(72^{\circ}\right)\right) \simeq 4 \times 10^{13}$ molecules $/ \mathrm{cm}^{2}$. STM measurements were performed at $4.8 \mathrm{~K}$ in the constant current mode using electrochemically etched polycrystalline $\mathrm{W}$ tips.

\section{RESULTS AND DISCUSSION}

\section{A. Isolated phthalocyanines}

We first investigate the structural and electronic properties of isolated Pc molecules. We considered the two typical semiconducting phthalocyanines: $\mathrm{CuPc}$ (Fig. 1, top) for $p$ type and $\mathrm{F}_{16} \mathrm{CuPc}$ (Fig. 1, bottom) for $n$-type semiconductors adopted in organic solar cells. The only difference of the two $\mathrm{Pc}$ molecules is that all $\mathrm{H}$ atoms in phenyl group of $\mathrm{CuPc}$ are substituted by $\mathrm{F}$ atoms to form $\mathrm{F}_{16} \mathrm{CuPc}$. Both molecules are planar structures and have four-fold symmetry. The average bond length of the four $\mathrm{Cu}-\mathrm{N}$ bonds is $1.944 \AA$ in $\mathrm{CuPc}$ and $1.946 \AA$ in $\mathrm{F}_{16} \mathrm{CuPc}$. The former is in agreement with the experimental value of $1.935 \AA$ with a standard deviation of $0.005 \AA$, as obtained from the x-ray diffraction measurements. ${ }^{48}$ The diagonal distance between the two outmost $\mathrm{F}$ atoms in $\mathrm{F}_{16} \mathrm{CuPc}$ is $15.47 \AA$, $0.43 \AA$ longer than

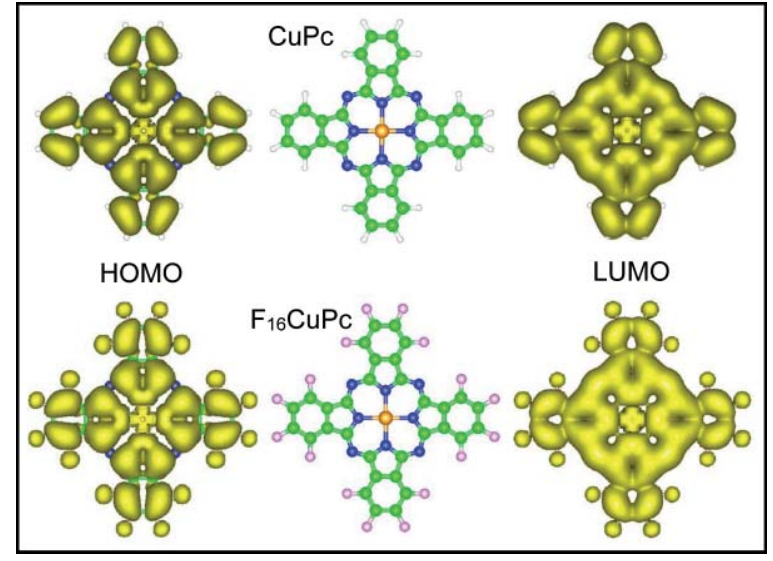

FIG. 1. Atomic structures of the isolated $\mathrm{CuPc}$ (top panel) and $\mathrm{F}_{16} \mathrm{CuPc}$ (bottom panel) molecules, and the corresponding charge density contour at 0.001 $e / \AA^{3}$ for the HOMO and LUMO states. C, H, F, N, and $\mathrm{Cu}$ atoms are denoted as green, white, pink, blue, and orange spheres, respectively.

the relevant $\mathrm{H}-\mathrm{H}$ distance in $\mathrm{CuPc}(15.04 \AA)$, because C-F bonds $(1.33 \AA$ ) are longer than $\mathrm{C}-\mathrm{H}$ bonds $(1.11 \AA)$. Figure 1 shows the electron density of the highest occupied molecular orbitals (HOMO) and lowest unoccupied molecular orbitals (LUMO) for the $\mathrm{CuPc}$ and $\mathrm{F}_{16} \mathrm{CuPc}$ molecules. For both molecules, there is a hole in the HOMO center, and the electron distribution is localized in the four pyrrole-like subunits and phenyl rings (Fig. 1, left panels). A small amount of electrons are distributed on all $\mathrm{F}$ atoms in the F-substituted phenyl group of $\mathrm{F}_{16} \mathrm{CuPc}$ molecule because of the large electron affinity of F. The LUMO (right panels of Fig. 1) are delocalized and distributed on the whole molecule. The major difference between the HOMO and LUMO charge densities is that the $\mathrm{N}$ atoms on the periphery of the square do not contribute to the former but have a major contribution to the latter for both molecules. It is worth pointing out that the LUMO of $\mathrm{F}_{16} \mathrm{CuPc}$ has more charge at the central $\mathrm{Cu}$ position than the HOMO; CuPc does not show obvious contrast between HOMO and LUMO at the central $\mathrm{Cu}$ position. This is consistent with experimental STM images. ${ }^{30}$

Since they serve as major light-absorbers in organic solar cells, we calculate the optical properties of these two molecules using TDDFT based on linear response; the absorbance spectra are shown in Fig. 2. The optical spectra not only help us to understand their sunlight-harvesting properties but also provide a more accurate description of the electronic structure, for instance, by correcting the underestimated band-gaps in LDA. In Fig. 2, it is evident that both Pc molecules exhibit two major absorption peaks (Q-band and $\mathrm{B}$-band) in the UV-visible light region. For CuPc, the first peak (Q-band) is at $624 \mathrm{~nm}$ and the second one (B-band) at $328 \mathrm{~nm}$ with a shoulder at $594 \mathrm{~nm}$. The shoulder is weak and less prominent than other absorption features but could be enhanced if the spectrum resolution is improved. Nevertheless, the obtained absorption spectrum is in good agreement with the experimentally measured UV-visible spectra, with the Q-band at $657 \mathrm{~nm}$, the B-band at $325 \mathrm{~nm}$, and a small shoulder at $600 \mathrm{~nm} \cdot{ }^{49}$ In addition, our results show small absorption peaks at $399 \mathrm{~nm}$ and $356 \mathrm{~nm}$ between the Q and B 


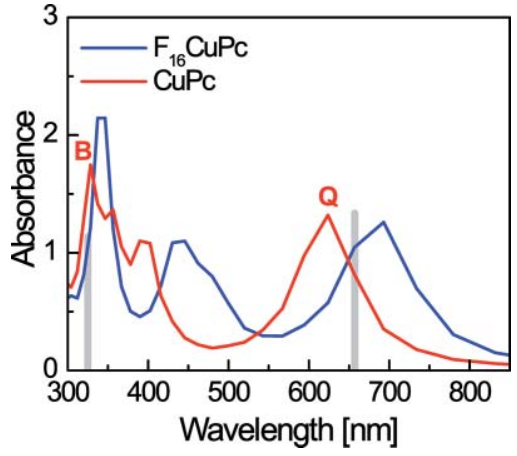

FIG. 2. UV-visible spectra of $\mathrm{CuPc}$ and $\mathrm{F}_{16} \mathrm{CuPc}$ molecules calculated from TDDFT. The two vertical gray lines show the absorption position and rescaled amplitudes of CuPc in the experimental spectrum (Ref. 49). The letters denote the absorption bands of $\mathrm{CuPc}$ (see text for details).

bands, which are missing in experiment because of the resolution limit. For the $\mathrm{F}_{16} \mathrm{CuPc}$ molecule, three absorbance bands show up at $693 \mathrm{~nm}, 446 \mathrm{~nm}$, and $346 \mathrm{~nm}$, respectively. Apparently, they are red-shifted from the corresponding values for CuPc. The red-shift of $0.2 \mathrm{eV}$ of the Q-band is consistent with the band-gap narrowing observed in optical measurements going from $\mathrm{CuPc}(1.7 \mathrm{eV})$ to $\mathrm{F}_{16} \mathrm{CuPc}$ $(1.5 \mathrm{eV}) .{ }^{15} \mathrm{~A}$ red-shift of $0.23 \mathrm{eV}$ is also observed in $\mathrm{CuPc}$ and $\mathrm{F}_{16} \mathrm{CuPc}$ thin-films despite the presence of intermolecular interactions. ${ }^{50}$

To elucidate the nature of the absorption bands, we analyze the electronic structure of both Pc molecules. The electronic energy levels as well as typical wavefunctions of $\mathrm{F}_{16} \mathrm{CuPc}$ are presented in Fig. 3. In LDA, the HOMO states are composed of two degenerate $a_{1 u}$ orbitals (combination of $2 p_{z}$ orbitals of $\mathrm{C}$ and $\mathrm{F}$ atoms), and a $b_{1 g \uparrow}$ orbital (combination of $3 d_{x^{2}-y^{2}}$ orbitals of $\mathrm{Cu}$ atom and $2 s, 2 p_{x}$, and $2 p_{y}$ orbitals of $\mathrm{N}$ atoms). The LUMO states are composed of one $b_{1 g}$ orbital with the same symmetry as $b_{1 g \uparrow}$, and four degenerate $e_{g}$ orbitals (combination of $2 p_{z}$ orbitals of $\mathrm{C}, \mathrm{N}$, and $\mathrm{F}$ atoms, and $3 d_{y z}$ and $3 d_{x z}$ orbitals of $\mathrm{Cu}$ atoms). Therefore, the HOMOs have mainly $\pi$ bonding character, while in the

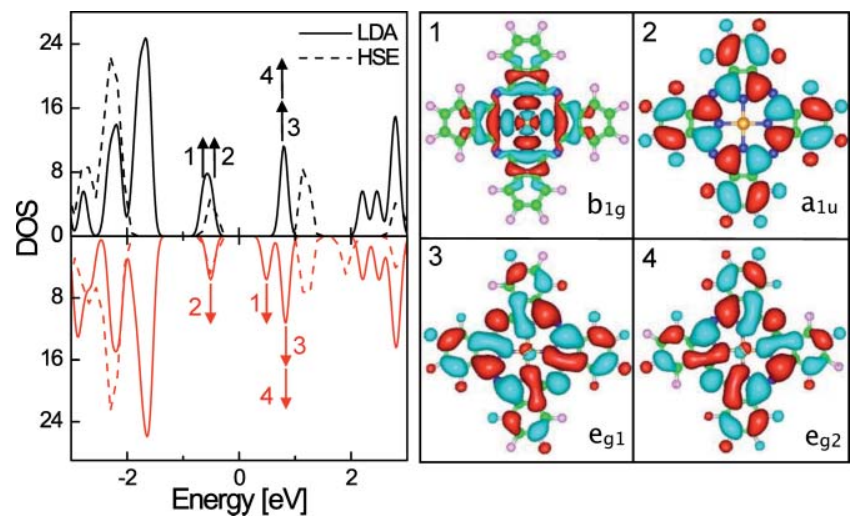

FIG. 3. Density of states (DOS) of the $\mathrm{F}_{16} \mathrm{CuPc}$ molecule: solid lines show the LDA results and dashed lines show the corresponding HSE results. Black lines and red lines (upper and lower panels) denote the spin-up and spin-down components, respectively. The Fermi level is set to zero. Wavefunctions for the HOMO and LUMO states are also displayed on the right, and labeled according to their symmetry. The labels (1)-(4) identify the positions of these states in the DOS diagram.
LUMOs the antibonding combination dominates. In comparison with $\mathrm{F}_{16} \mathrm{CuPc}$, the $\mathrm{HOMO}$ and LUMO orbitals of $\mathrm{CuPc}$ have similar symmetry without electron distribution on the $\mathrm{F}$ atoms, consistent with previous calculations. ${ }^{47}$ The Q-band at $624 \mathrm{~nm}$ for $\mathrm{CuPc}$ and $693 \mathrm{~nm}$ for $\mathrm{F}_{16} \mathrm{CuPc}$ is attributed to electronic excitations from HOMO to LUMO of the molecule, and hence represents a $\pi \rightarrow \pi^{\star}$ transition. Furthermore, the electronic band gap of $\mathrm{F}_{16} \mathrm{CuPc}$ is around $0.2 \mathrm{eV}$ smaller than that of $\mathrm{CuPc}$, which accounts for the red-shift in UV-visible spectra discussed earlier.

In order to obtain more accurate band alignments, we also use the HSE functional (from the VASP code) to calculate the density of states (DOS) for the system. Although producing the same main features, the HSE functional modifies the position of localized molecular orbitals obtained from LDA calculations. In particular, it moves the $b_{1 g \uparrow}$ orbital of $\mathrm{F}_{16} \mathrm{CuPc}$ away from $a_{1 u}$ orbitals to mix with the states of lower energy, while it moves the $b_{1 g \downarrow}$ orbital up in energy, now located $0.7 \mathrm{eV}$ above the $e_{g}$ LUMO states. Similar trends apply to CuPc. ${ }^{47}$ The discrepancy between LDA and HSE results lies in the inaccurate description of localized states $\left(b_{1 g}\right)$ in LDA, which contains a large self-interaction error for such states. The HSE functional, by incorporating a fraction of exact exchange at short distances, largely removes selfinteraction errors and corrects the energy of localized orbitals. Consequently, for more delocalized $a_{1 u}$ and $e_{g}$ orbitals, which are dominant in the HOMO and LUMO states, HSE and LDA yield very close energies due to smaller self-interaction errors. Accordingly, LDA results for the frontier states in the Pc layers on graphene should be reasonable, since those states will be more delocalized due to intermolecular interactions and molecule-substrate interaction.

\section{B. Experimental observations}

When $\mathrm{F}_{16} \mathrm{CuPc}$ molecules are deposited at room temperature onto epitaxial graphene grown on $\mathrm{SiC}(0001)$, wellordered molecular self-assembly patterns form. Figures 4 and 5 show measured STM images of the $\mathrm{F}_{16} \mathrm{CuPc} / \mathrm{EG}$ adsorption system. MEG and BEG coexists on the SiC substrate,

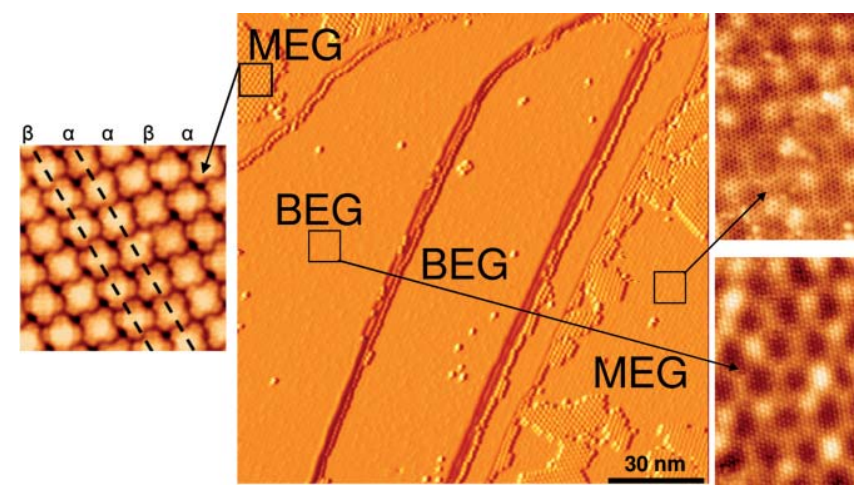

FIG. 4. Scanning tunneling microscopy (STM) images for $\mathrm{F}_{16} \mathrm{CuPc}$ molecules adsorbed on monolayer epitaxial graphene (MEG) and bilayer epitaxial graphene (BEG). Selective adsorption on MEG is evident. Characteristic STM images for the MEG, BEG,s and $\mathrm{F}_{16} \mathrm{CuPc}$ layers are also shown on the sides, enlarged. 
whose STM images in Fig. 4 agree well with features from other measurements of MEG and BEG in the literature. ${ }^{51,52}$ Namely, although both demonstrate a $(6 \times 6)$ superstructure, MEG shows a honeycomb structure while BEG displays a triangular grid feature. ${ }^{51}$ Careful inspection of Fig. 4 clearly shows that $\mathrm{F}_{16} \mathrm{CuPc}$ molecules prefer to adsorb on MEG rather than BEG at low coverage: they form ordered closepacked islands on the former while only scattered molecules are found on the latter. Inside the islands, the molecules are well-ordered and arranged into hexagonal-like patterns, with hundreds of parallel rows (stripes) of $\mathrm{F}_{16} \mathrm{CuPc}$ of the same orientation (dashed lines in Fig. 4, left panel). The pattern has lattice constants of $15.1 \AA \times 16.0 \AA$ at an angle of $72^{\circ}$. Detailed investigation shows the direction of the molecular rows is roughly along the $\mathrm{C}-\mathrm{C}$ bond direction (the armchair direction) of the underlying graphene substrate. Only two molecular orientations appear among these rows with the molecular diagonal forming $\sim 60^{\circ}$ and $27^{\circ}$ angles with the row-axis. They are named as $\alpha$ and $\beta$ stripes, respectively. From Fig. 4, we observe that the alternating $\alpha \beta$ arrangement is dominant (referred to as $\alpha \beta$ phase), while there exist places where $\alpha$-rows are neighboring each other (referred to as $\alpha \alpha$ phase). Comparing them with the simulated STM images of single $\mathrm{F}_{16} \mathrm{CuPc}$, we found that the electron density in the LUMOs of $\mathrm{F}_{16} \mathrm{CuPc}$ is in excellent agreement with the experimental measurement (see Fig. 5). Previous STM studies ${ }^{37}$ on $\mathrm{CuPc}$ adsorption on graphite show a different pattern for $\mathrm{CuPc}$ self-assembly, namely a square lattice with dimensions of 13.8 $\AA \times 13.8 \AA$. This is comparable with the molecular size of $\mathrm{CuPc}$ discussed above.

\section{Uniform phthalocyanine overlayers}

We study next the adsorption of Pc on graphene using our theoretical approach. We start from uniform phases of $\mathrm{F}_{16} \mathrm{CuPc}$ and $\mathrm{CuPc}$ overlayers, that is, adsorption of a single molecule per unit cell on the graphene substrate. This is equivalent to simulating the $\alpha \alpha$ stripes for $\mathrm{F}_{16} \mathrm{CuPc}$ and the square lattice for $\mathrm{CuPc}$. To match the experimental lattice dimensions of $15.1 \AA \times 16.0 \AA$ observed in the STM image

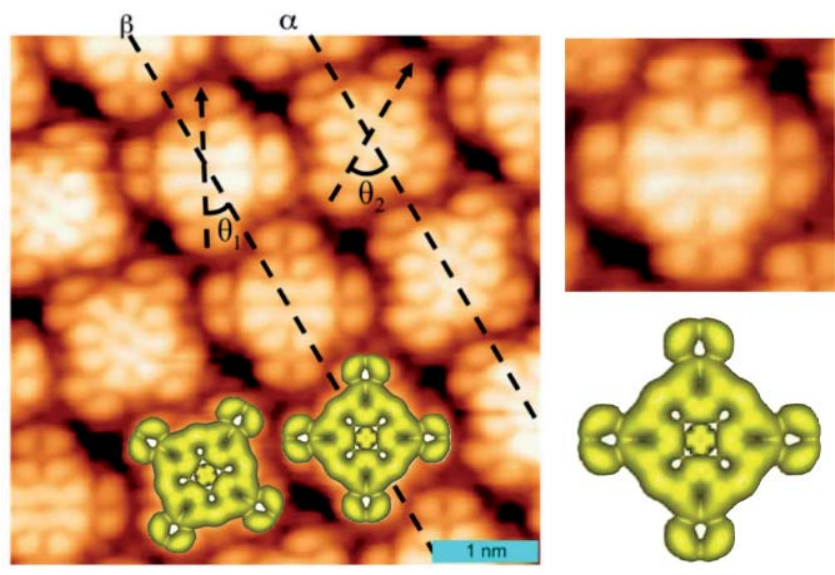

FIG. 5. High-resolution STM images of ordered $\mathrm{F}_{16} \mathrm{CuPc}$ patterns on epitaxial graphene. Simulated STM image for $\mathrm{F}_{16} \mathrm{CuPc}$ from the LUMO charge density is shown side-by-side (right panel) and overlaid onto the experimental images (left panel). in Fig. 4, we consider two possible supercells: in the first, the supercell lattice vectors run along the armchair direction of graphene (denoted as gra[(3,4) $\times(4,3)]$ ), while in the second the lattice vectors are along the zigzag direction (denoted as $\operatorname{gra}[(6,0) \times(6,1)])$; the former is closer to what is observed in experiment.

First, we vary the adsorption height and orientation of $\mathrm{F}_{16} \mathrm{CuPc}$ with respect to the underlying graphene lattice to find the optimal structure. Within the gra[ $[3,4) \times(4,3)]$ supercell, the minimum-energy structure is obtained when $\mathrm{F}_{16} \mathrm{CuPc}$ is located 3.08 $\AA$ above the EG surface and the azimuthal angle is $-9^{\circ}$, defined as the angle between the molecular diagonal, coincident with the N-Cu-N axis, and the short diagonal direction of the unit cell, shown in Fig. 6(a). This orientation is very close to the experimental observation in Fig. 5. Similarly, the optimized geometry in the gra[ $[6,0) \times(6,1)]$ supercell has an adsorption height of $3.05 \AA$ and azimuthal angle of $-19^{\circ}$. In both cases, the central $\mathrm{Cu}$ atom is situated directly above the site of a $\mathrm{C}$ atom in EG structure. Upon adsorption on graphene, the molecular structure is slightly changed: the adsorbed molecule deviates from the ideal planar structure, with the $\mathrm{N}$ atoms around $\mathrm{Cu}$ being $0.04 \AA$ higher and the periphery $\mathrm{F}$ atoms $0.10 \AA$ lower than the central $\mathrm{Cu}$ atom. The average $\mathrm{Cu}-\mathrm{N}, \mathrm{N}-\mathrm{C}$, and $\mathrm{C}-\mathrm{F}$ bond lengths are $1.937 \AA, 1.347$ $\AA$, and $1.327 \AA$, respectively, compared to the corresponding values of $1.946 \AA, 1.349 \AA$, and $1.328 \AA$ in the gas phase. However, the molecule is slightly distorted as indicated by a shorter $\mathrm{Cu}-\mathrm{N}$ bond length of $1.904 \AA$ in one diagonal direction and a longer $\mathrm{Cu}-\mathrm{N}$ bond length of $1.971 \AA$ in the other direction, which is due to geometric constraints imposed by the artificial periodic boundary conditions. As a result, the molecular size shrinks by $0.27 \AA$ in the former diagonal direction. The adsorption energy is defined as

$$
E_{a}=\left(E_{t o t}-E_{g r a}-n \times E_{P c}\right) / n
$$

where $E_{t o t}, E_{g r a}$, and $E_{P c}$ is the total energy of the adsorption system, the energy of the isolated graphene substrate, and the energy of gas-phase Pc molecule, respectively, and $n$ is the number of Pc molecules in the unit cell. The adsorption energies are $E_{a}=-3.19 \mathrm{eV}$ and $-3.17 \mathrm{eV}$ in the $\operatorname{gra}[(3,4) \times(4,3)]$ and $\operatorname{gra}[(6,0) \times(6,1)]$ supercells, respectively, using vdW-DF functionals at adsorption height of $\sim 3.4 \AA$. The interaction energy of $56 \mathrm{meV}$ per atom is in the same energy range of 35-60 meV/atom for graphite exfoliation indicating that the Pc-graphene interaction is mainly a dispersion force. ${ }^{53}$ Since the relative orientation of the Pc overlayer with respect to graphene in $\operatorname{gra}[(3,4) \times(4,3)]$ is closer to the pattern observed in experiments, ${ }^{34}$ we focus on this configuration in the following.

Figure 6(b) shows a contour plot of the adsorption potential energy surface of a single $\mathrm{F}_{16} \mathrm{CuPc}$ molecule on graphene in the gra $[(3,4) \times(4,3)]$ unit cell. The area of the energy contours corresponds to the black rectangular region marked in Fig. 6(a). The dark blue region at $(1.42,0) \AA$ and $(0.71$, 1.23) $\AA$ is both close to the site of a carbon atom in graphene, and correspond to the highest binding energy of $3.19 \mathrm{eV}$ indicating the stability of this site for $\mathrm{F}_{16} \mathrm{CuPc}$ adsorption on EG. Furthermore, between these two stable sites, Fig. 6(b) reveals a smooth diffusion pathway with a very small barrier 
(a)

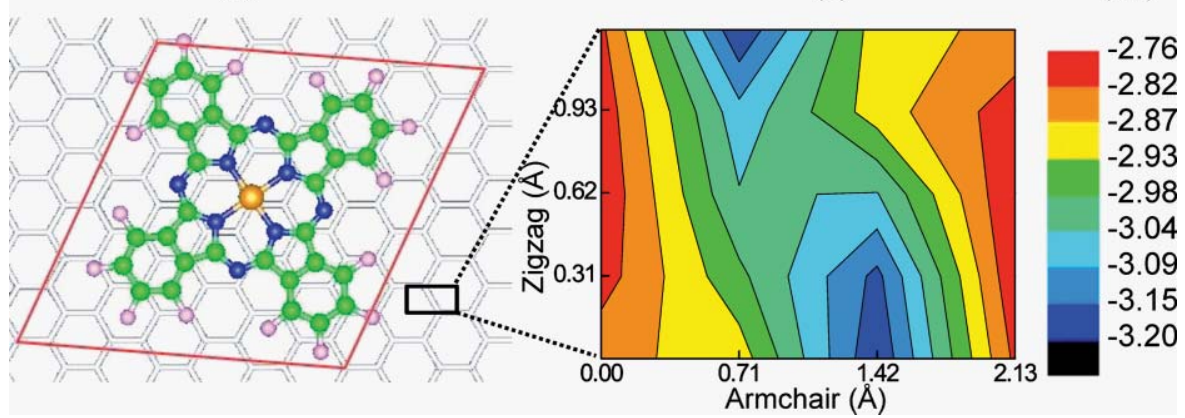

FIG. 6. (a) The optimized configuration and (b) potential energy surface contours for the $\mathrm{F}_{16} \mathrm{CuPc} / \mathrm{gra}[(3,4) \times(4,3)]$ system. Graphene is shown as a gray sheet to distinguish it from $\mathrm{C}$ atoms in the Pc molecule. The unit cell is described by a red parallelogram. The potential energy surface is calculated by using a (4×5) grid sampling in the rectangular region marked in the lower right corner of (a).

of $\sim 0.16 \mathrm{eV}$ for $\mathrm{F}_{16} \mathrm{CuPc}$ on EG. Hence the molecule could diffuse freely on EG to find its most stable adsorption site.

On the basis of the optimized configuration, we then investigated the electronic structure of $\mathrm{F}_{16} \mathrm{CuPc} / \mathrm{gra}[(3,4) \times(4,3)]$. The corresponding DOS of the adsorption system (thick black solid line) as well as that projected onto the Pc molecule (blue solid line) and the graphene substrate (thin black solid line) are presented in Fig. 7. The two features below and above $E_{F}$ in the total DOS mainly come from the contributions of HOMO and LUMO states of $\mathrm{F}_{16} \mathrm{CuPc}$, which comprises two spin-up and a spin-down orbital (for HOMOs), and two spin-up and three spin-down orbitals (for LUMOs), respectively, as described in Sec. III A. The HOMO and LUMO states are $0.93 \mathrm{eV}$ and $0.37 \mathrm{eV}$ below and above $E_{F}$, respectively, producing a gap of $1.30 \mathrm{eV}$, which is smaller than the experimental value (1.5-1.8 eV) (Ref. 15) and the HSE band-gap (1.7 eV); the band-gap underestimation is typical in LDA. More importantly, LUMO states of Pc in the adsorption system have a non-zero occupation indicating a small amount of electron transfer from graphene to $\mathrm{F}_{16} \mathrm{CuPc}$. This is also consistent with the fact that $E_{F}$ is $40 \mathrm{meV}$ lower than the Dirac point of EG.

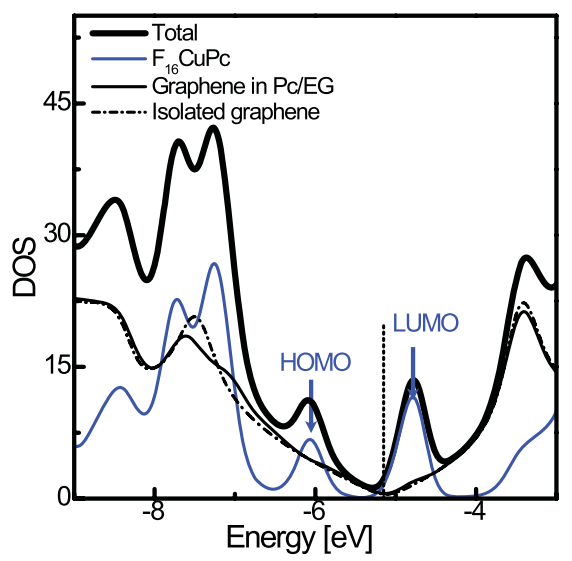

FIG. 7. Total DOS (thick black solid line) and projected DOS on the Pc molecule (blue solid line), on graphene (thin black solid line), upon $\mathrm{F}_{16} \mathrm{CuPc}$ adsorption on graphene, and the DOS of the isolated graphene (black dashed dotted line). The vertical dotted line shows the Fermi level.
In order to elucidate modifications in the graphene electronic structure upon $\mathrm{F}_{16} \mathrm{CuPc}$ adsorption, we compare the projected DOS (PDOS) on graphene in the adsorption system with that of isolated graphene (black dashed dotted line), also shown in Fig. 7. The PDOS of EG shows two small features at $0.65 \mathrm{eV}(0.39 \mathrm{eV})$ below (above) the $E_{D}$, and a major smoothing effect for the peak in the range of $2-3 \mathrm{eV}$ below $E_{D}$. This suggests that the electronic states of graphene are redistributed upon $\mathrm{F}_{16} \mathrm{CuPc}$ adsorption. Experimentally, an additional peak at $0.4 \mathrm{eV}$ above $E_{D}$ is observed in scanning tunneling spectroscopy (STS) of graphene with adsorbed molecules ${ }^{34}$ consistent with the features in Fig. 7.

To gain more insight into the electronic interaction between $\mathrm{F}_{16} \mathrm{CuPc}$ and graphene, we show in Fig. 8 the bonding character and charge redistribution upon $\mathrm{F}_{16} \mathrm{CuPc}$ adsorption by calculating the corresponding charge density differences (CDD), defined as,

$$
\Delta \rho=\rho_{t o t}-\rho_{P c}-\rho_{g r a}
$$

where $\rho_{t o t}, \rho_{P c}$, and $\rho_{g r a}$ are the charge density of the combined system, isolated Pc molecule, and graphene with geometries fixed at the optimized ones in adsorption system. From the calculated CDD, it is clear that $p_{z}$ orbitals of the Pc molecule and EG are mainly involved (Fig. 8, top-right panel). Furthermore, the charge redistribution generates an additional layer of electrons between Pc and EG, yielding a dipole layer pointing towards the Pc molecule. Integrating the charge density difference along the surface normal direction $z$ suggests a net amount of $0.08 e$ charge transferred from $\mathrm{EG}$ to $\mathrm{F}_{16} \mathrm{CuPc}$. The majority of transferred charge is distributed around the central $\mathrm{Cu}$ atom, the interfacial region below $\mathrm{Cu}$ atom, and the F-substituted phenyl rings on the molecular periphery. Meanwhile, electron depletion of $p_{z}$ orbitals and accumulation in $\sigma^{\star}$ orbitals in EG beneath the phenyl rings indicate a charge redistribution in EG in the presence of $\mathrm{F}_{16} \mathrm{CuPc}$, which explains the changes in the graphene PDOS displayed in Fig. 7.

The Dirac point is $0.39 \mathrm{eV}(0.55 \mathrm{eV})$ below the $E_{F}$ for BEG (MEG) in STS, ${ }^{34}$ which indicates a charging effect from $\mathrm{SiC}$ to $\mathrm{EG}$. A charged graphene single-layer with $0.005 e(0.01 e)$ per carbon atom would have a higher $E_{F}$ by $0.4 \mathrm{eV}$ and $0.6 \mathrm{eV}$ from that of the neutral system (where $E_{F}$ 


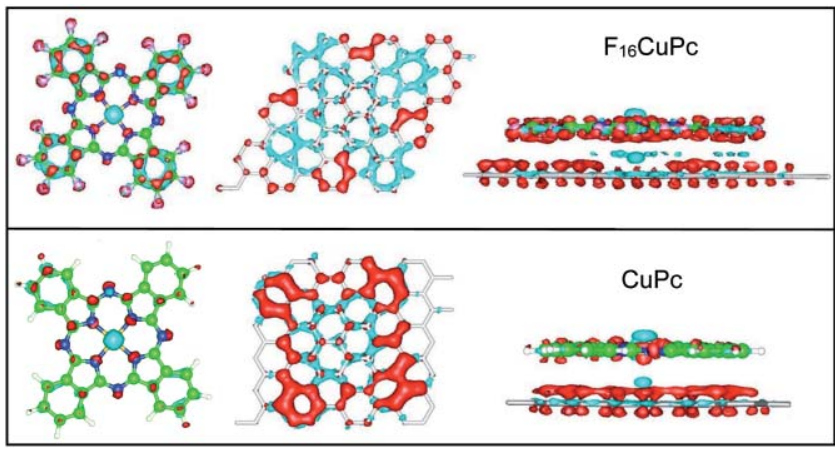

FIG. 8. Top view and side view of the charge density difference for $\mathrm{F}_{16} \mathrm{CuPc} / \mathrm{gra}[(3,4) \times(4,3)]$ (top panel) and $\mathrm{CuPc} / \mathrm{gra}[(1,5) \times(4,3)]$ (bottom panel) at contour levels of $0.003 e / \AA^{3}$. The light blue and red clouds correspond to regions with electron accumulation and depletion, respectively.

and $E_{D}$ coincide). Therefore, we charge the $\operatorname{gra}[(3,4) \times(4,3)]$ unit cell, which contains 80 carbon atoms, by $0.4 e$ and $0.8 e$, respectively, to model the realistic electronic conditions of BEG and MEG, representing the substrate charging effect of $\mathrm{SiC}$. The relevant $E_{F}$ shift with respect to the charges applied in the system is presented in Table I. With the increase of charge, $E_{F}$ gradually rises from $-0.03 \mathrm{eV}$ below $E_{D}$ (neutral) to $0.34 \mathrm{eV}$ above $E_{D}$ ( $-0.8 e$ charged), while the LUMO of Pc shifts only slightly towards higher energy with respect to $E_{D}$. Consequently, more electrons are transferred to the Pc molecule from the charged EG. The PDOS of EG, however, is not influenced by the added charge. By integrating the charge density difference along the surface normal direction, we find a net charge transfer of $0.32 e(0.57 e)$ from BEG (MEG) to $\mathrm{F}_{16} \mathrm{CuPc}$. Obviously, the negatively charged system contributes to enhanced electron transfer and electron accumulation in Pc molecules.

In Table I, we also summarize the amount of charge transfer from $\mathrm{EG}$ to $\mathrm{F}_{16} \mathrm{CuPc}$ as a function of the thickness of epitaxial graphene layers and the corresponding voltage applied. The table shows that, with the increase of EG thickness on $\mathrm{SiC}$ or the decrease of negative voltage, the charge transfer from graphene to physisorbed Pc can be continuously tuned. This provides a simple method for controlling electron doping into the organic conduction layer and the graphene at the interface, avoiding the complications associated with chemical reactions in chemical doping. The nonlinear trend further indicates that this doping method, by varying layer thickness and voltage in the physisorption system, is nontrivial. For

TABLE I. The calculated Fermi-level shift $\left(\Delta E_{F}\right)$ with respect to the Dirac point of graphene and the corresponding charge transfer $(\Delta q)$ in the neutral and charged systems. The Dirac point is set to zero. $\mathrm{N}_{E G}$ denotes the thickness of epitaxial graphene layers.

\begin{tabular}{lcccrr}
\hline \hline & & & & \multicolumn{2}{c}{$\Delta q(e)$} \\
\cline { 5 - 6 } Experiment $(\mathrm{V})$ & Theory $(e)$ & $\mathrm{N}_{E G}$ & $\Delta E_{F}(\mathrm{eV})$ & $\mathrm{F}_{16} \mathrm{CuPc}$ & $\mathrm{CuPc}$ \\
\hline-0.6 & -0.8 & 1 & 0.34 & 0.57 & 0.59 \\
-0.4 & -0.4 & 2 & 0.25 & 0.32 & 0.38 \\
0 & 0 & $\ldots$ & -0.03 & 0.08 & 0.14 \\
$\ldots$ & 0.4 & $\ldots$ & $\ldots$ & -0.06 & -0.06 \\
\hline \hline
\end{tabular}

completeness, we also include a case of positively charged graphene, which can be achieved by applying positive voltages on the graphene layer or depositing graphene onto substrates with higher electron affinity. ${ }^{52}$ We find a reverse electron transfer of $0.06 e$ from $\mathrm{F}_{16} \mathrm{CuPc}$ to graphene when a $0.4 \mathrm{~V}$ positive bias is applied.

The tunable electronic coupling between $\mathrm{F}_{16} \mathrm{CuPc}$ and graphene by charge transfer results in differences in adsorption structure. An interesting phenomenon observed in the STM images (Fig. 4) is that ordered molecular islands are flat and distributed on MEG rather than on BEG at low coverages. This suggests two effects: (i) the molecule-substrate interaction is stronger than the intermolecular interaction and (ii) the interaction between the Pc molecule and MEG is stronger than that of Pc and BEG. The selective adsorption behavior of $\mathrm{F}_{16} \mathrm{CuPc}$ on MEG can be attributed to the fact that the local DOS of MEG, with $E_{F}$ being $0.16 \mathrm{eV}$ higher than $E_{D}$, is larger than that of BEG. Consequently, the stronger electronic coupling of Pc and MEG explains the distribution of ordered Pc islands on MEG. Our DFT calculations also show that the adsorption energy of $\mathrm{F}_{16} \mathrm{CuPc}$ on MEG and BEG (modeled by the charged substrate explained above) is $3.93 \mathrm{eV}$ and $3.54 \mathrm{eV}$, respectively. The energy difference of $0.39 \mathrm{eV}$, together with the small diffusion barrier $(0.16 \mathrm{eV})$, indicates that the Pc molecule prefers to adsorb on MEG when deposited at room temperature. This explains the experimental observation ${ }^{34}$ that no Pc is found on BEG at low coverage $(<0.4 \mathrm{ML})$. Another observation is that $\mathrm{F}_{16} \mathrm{CuPc}$ molecules prefer to form a flat monolayer rather than multilayered islands on EG. To understand the energetically favorable molecular monolayers, we calculate the stacking energy for a pair of $\mathrm{F}_{16} \mathrm{CuPc}$ molecules with the top $\mathrm{Pc}$ rotated by $45^{\circ}$ with respect to the bottom one and at a distance of $3.18 \AA$. This configuration produces the largest stacking energy equal to $2.46 \mathrm{eV}$. This stacking energy is significantly lower than the adsorption energy per molecule on MEG, by more than $1.0 \mathrm{eV}$, which makes the stacked structure unstable.

For comparison, we also studied the adsorption of CuPc on graphene. Figure 9(a) represents the calculated atomic structure of the $\mathrm{CuPc}$ layers on graphene using the unit cell of $[(1,5) \times(4,3)]$. The lattice of adsorbed $\mathrm{CuPc}$ overlayer is almost square with dimensions of $13.69 \AA \times 14.96 \AA$. The measured lattice constant in experiments $\mathrm{s}^{31,37}$ ranges from $13.8 \AA$ to $15.0 \AA$. The CuPc overlayer on graphene is more compact than that of $\mathrm{F}_{16} \mathrm{CuPc}$ because of the smaller molecular size (diagonal H-H distance of $15.04 \AA$ for CuPc vs. diagonal F-F distance of $15.47 \AA$ for $\mathrm{F}_{16} \mathrm{CuPc}$ ) and weaker $\mathrm{H}-\mathrm{H}$ repulsion than F-F repulsion between neighboring molecules. The most stable adsorption configuration is still one in which the central $\mathrm{Cu}$ atom of $\mathrm{Pc}$ is situated directly above the site of a $\mathrm{C}$ atom of graphene, similar to $\mathrm{F}_{16} \mathrm{CuPc}$ adsorption on graphene. The optimal orientation of CuPc in Fig. 9 is characterized by the angle between the molecular diagonal direction and the supercell lattice vector, which is $27^{\circ}$, in good agreement with the value $24^{\circ}$ measured in experiment. ${ }^{31,37}$ The adsorption height is $3.15 \AA$ in LDA and the binding energy is $2.47 \mathrm{eV}$ from the vdW-DF calculation (height $\sim 3.4 \AA$ ), which is $0.72 \mathrm{eV}$ lower than that of $\mathrm{F}_{16} \mathrm{CuPc} / \mathrm{gra}[(3,4) \times(4,3)]$. The smaller absorption energy of $\mathrm{CuPc}$ than $\mathrm{F}_{16} \mathrm{CuPc}$ is attributed to weaker 

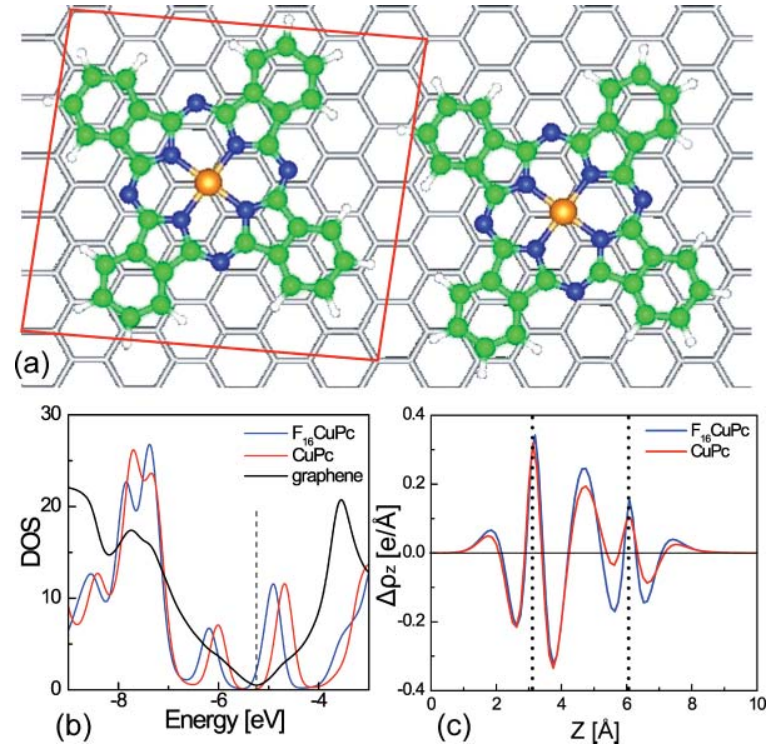

FIG. 9. (a) Geometry of CuPc molecules adsorbed on graphene[(1,5) $\times(4,3)]$. (b) Corresponding density of states (DOS) plot projected on $\mathrm{CuPc}$ and graphene for the adsorption system, respectively. The vertical dashed line marks the Dirac point which is $10 \mathrm{meV}$ below the Fermi level for $\mathrm{CuPc} /$ graphene. (c) One-dimensional charge density difference along the surface normal direction of graphene; vertical dotted lines mark the positions of graphene and the molecule planes. For comparison, the corresponding lines of $\mathrm{F}_{16} \mathrm{CuPc}$ (blue) are also shown in panels (b) and (c).

van der Waals interaction between $\mathrm{CuPc}$ and graphene and between $\mathrm{CuPc}$ molecules, due to the smaller charge density around the periphery $\mathrm{H}$ atoms than $\mathrm{F}$ atoms in $\mathrm{F}_{16} \mathrm{CuPc}$, and the larger distance between $\mathrm{CuPc}$ and graphene by $0.07 \AA$. Recently, it was proposed that the interaction between an anion and substituted $\pi$ system is simply a charge-dipole interaction, ${ }^{54}$ which might be used to explain the enhanced binding of $\mathrm{F}_{16} \mathrm{CuPc}$ over $\mathrm{CuPc}$ on graphene.

The corresponding DOS and one-dimensional charge difference plot is shown in panels (b) and (c) of Fig. 9, together with those of $\mathrm{F}_{16} \mathrm{CuPc}$ for direct comparison. We found that the DOS features are qualitatively close to those of the $\mathrm{F}_{16} \mathrm{CuPc} / g r a p h e n e$ system. The biggest difference between the two cases is the level alignment: with reference to the Dirac point of graphene $E_{D}$, the HOMO of CuPC is $0.76 \mathrm{eV}$ below $E_{D}$ while the $\mathrm{HOMO}$ of $\mathrm{F}_{16} \mathrm{CuPc}$ is $0.94 \mathrm{eV}$ below $E_{D}$, and the LUMO is $0.56 \mathrm{eV}$ and $0.34 \mathrm{eV}$ higher than $E_{D}$ for $\mathrm{CuPc}$ and $\mathrm{F}_{16} \mathrm{CuPc}$, respectively. Since the LUMO of $\mathrm{F}_{16} \mathrm{CuPc}$ is closer to the Fermi level, it could facilitate easier charge transfer from graphene to the molecule consistent with its $n$-type nature. The LUMO of CuPc is higher in energy and, hence, it is more difficult to accept electrons from the substrate enhancing its $p$-type character. Our result is in good agreement with photoelectronic measurements of the HOMO position on HOPG: ${ }^{55}$ it is $0.9 \mathrm{eV}$ and $1.2 \mathrm{eV}$ below the $E_{F}$ of $\mathrm{HOPG}$ for $\mathrm{CuPc}$ and $\mathrm{F}_{16} \mathrm{CuPc}$, respectively. The fact that the LUMO of $\mathrm{F}_{16} \mathrm{CuPc}$ is closer to the Fermi level than that of $\mathrm{CuPc}$ is also consistent with experimental observation that $\mathrm{F}_{16} \mathrm{CuPc}$ images (LUMO) are much brighter than $\mathrm{CuPc}$ under negative bias of $-2.0 \mathrm{~V},{ }^{30}$ which samples a higher electron density in this energy range. Similar to the case of $\mathrm{F}_{16} \mathrm{CuPc}$, the projected DOS on graphene also shows that its electronic structure is modified upon CuPc adsorption: the DOS is smoothed at the peak $2.28 \mathrm{eV}$ below the Dirac point and an extra DOS peak shows up $0.39 \mathrm{eV}$ above the Dirac point. Furthermore, the band gap of $\mathrm{F}_{16} \mathrm{CuPc}$ is $\sim 40$ meV smaller than that of CuPc.

The redistribution of electron density upon CuPc adsorption is also similar to the case of $\mathrm{F}_{16} \mathrm{CuPc}$. However, $\mathrm{F}_{16} \mathrm{CuPc}$ shows much higher electron polarization at the interface displayed by the higher positive peak between Pc and graphene planes and the lower negative peaks in the molecule region (Fig. 9(c)). The spacial distribution of polarized charge for $\mathrm{CuPc/graphene} \mathrm{is} \mathrm{shown} \mathrm{in} \mathrm{Fig.} 8$ (bottom panels). A significant difference from that for $\mathrm{F}_{16} \mathrm{CuPc}$ in Fig. 8 (top middle panel) is that the charge redistribution on CuPc is very small, while more electrons are located around the benzenelike rings and $\mathrm{F}$ atoms in $\mathrm{F}_{16} \mathrm{CuPc}$. There seems to be more electron loss in graphene upon $\mathrm{CuPc}$ adsorption characterized by large-area red clouds in Fig. 8 (bottom middle panel); however, this is compensated by an electron accumulation layer closer to EG in the present case (blue clouds). As a result, the plane-averaged net electron redistribution is similar to the case of $\mathrm{F}_{16} \mathrm{CuPc} /$ graphene. The slight difference in the exact numbers of charge transfer in Table I $(0.14 e$ for $\mathrm{CuPc}$ vs. $0.08 e$ for $\mathrm{F}_{16} \mathrm{CuPc}$ ) is mainly a result of artificial spacial partition by choosing a particular plane to separate $\mathrm{Pc}$ and graphene; due to the non-homogeneous charge redistribution, these numbers do not reflect the "true" electronegativity, neither the $p$ - or $n$-type behavior of two molecules of different composition and size. The amount of electron transfer from $\mathrm{CuPc}$ to graphene follows the same trend as that of $\mathrm{F}_{16} \mathrm{CuPc}$ with the increase of graphene voltage or the decrease of EG thickness, as shown in Table I. CuPc could gain $0.59 e$ and $0.38 e$ on MEG and BEG, respectively. The larger polarization effect at the $\mathrm{F}_{16} \mathrm{CuPc} / g r a p h e n e$ interface is consistent with the stronger electronegative properties of the $\mathrm{F}_{16} \mathrm{CuPc}$ molecule. As a result, the induced interface dipole for $\mathrm{F}_{16} \mathrm{CuPc}$ is greater than that for $\mathrm{CuPc}$, blocking electron transfer from $\mathrm{F}_{16} \mathrm{CuPc}$ to graphene in the former when graphene is used as transparent anodic electrode in photovoltaic devices. ${ }^{25}$

\section{Non-uniform $\boldsymbol{F}_{16} \mathrm{CuPc}$ stripes}

Next we simulate the non-uniform $\mathrm{F}_{16} \mathrm{CuPc}$ structure with molecular stripes of different orientations adsorbed on EG, namely, the $\alpha \beta$ phase. To this end, we construct a graphene supercell of $[(3,4) \times(8,6)]$, with two $\mathrm{F}_{16} \mathrm{CuPc}$ molecules, as shown in Fig. 10. The lattice vectors are $14.96 \AA$ and $29.92 \AA$, at an angle of $69.4^{\circ}$. In the patterns observed by STM, shown in Fig. 5, an incommensurate Pc overlayer with respect to the underlying graphene lattice implies a small lattice mismatch between the overlayer and substrate lattices, which would require a very large supercell. We are restricted to commensurate supercells by computational considerations, and accordingly we have identified the best matching supercell as $2 \mathrm{~F}_{16} \mathrm{CuPc} / \mathrm{gra}[(3,4) \times(8,6)]$. Figure 10 (a) represents the $\alpha \alpha$ phase, where the two molecules in the unit cell have the same orientation forming parallel rows. The patterns in Figs. 10(b)-10(d), are models for the $\alpha \beta$ phase with $\mathrm{F}_{16} \mathrm{CuPc}$ 


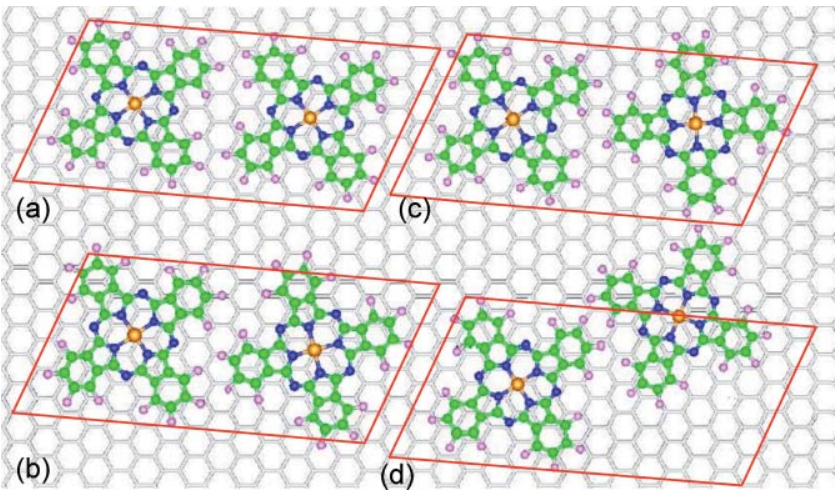

FIG. 10. Geometries of uniform and non-uniform $\mathrm{F}_{16} \mathrm{CuPc}$ overlayers adsorbed on graphene $[(3,4) \times(8,6)]$ : (a) the $\alpha-\alpha$ pattern; (b)-(d) the $\alpha-\beta$ stripes with relative azimuthal angles of: (b) $20^{\circ}$, (c) $30^{\circ}$ and (d) $40^{\circ}$. The unit cell (red parallelogram) contains two Pc molecules.

rows of two different orientations, corresponding to the $\alpha$ and $\beta$ stripes. The relative azimuthal angles for the two molecules in each unit cell in Figs. 10 (b) -10 (d) are: $20^{\circ}, 30^{\circ}$, and $40^{\circ}$, respectively. In addition, we also adjust the translational position of the second Pc molecule with respect to the first one after rotation, to minimize the steric repulsion between the two molecules.

The binding energy is $-6.16 \mathrm{eV} / \mathrm{UC}$ for the $\alpha \alpha$ orientation. The $\alpha \beta$ structures have very close total energies with a binding energy $-5.99 \pm 0.03 \mathrm{eV} / \mathrm{UC}$ for the different arrangements shown in Figs. 10(b)-10(d). The difference is within the limit of computational accuracy. We found that the structure in Fig. 10(c) has closest orientations of the molecules to those observed in experiment (Fig. 5). The different molecular orientation in $\alpha \beta$ phase results in a small energy shifts of the HOMO and LUMO values by less than $0.1 \mathrm{eV}$.

The small difference in adsorption energy of $-0.1 \mathrm{eV} / \mathrm{Pc}$ indicates that $\mathrm{Pc}$ molecules in the $\alpha \alpha$ and $\alpha \beta$ orientations are almost energetically degenerate though the $\alpha \alpha$ alignment is slightly favored within this artificial unit cell. When this periodic boundary condition is applied to a single layer of free-standing molecules (without the graphene substrate), the intermolecular interactions differ significantly in the two phases: they are overall attractive $(-0.27 \mathrm{eV})$ for $\alpha \alpha$ but repulsive $(+0.23 \mathrm{eV})$ for $\alpha \beta$. The F-F diagonal distance along the $\alpha \beta$ direction is compressed by $0.5 \AA$, due to the incommensurate more compact lattice used along this direction. To mimic the real dimensions observed in experiment, ${ }^{34}$ we use supercells where only the lattice vector along the $\alpha \beta$ direction is extended by $1 \AA$ in the free-standing layer of molecules. The supercell adjustment is applied to both the $\alpha \alpha$ and $\alpha \beta$ phases. The intermolecular interactions in both phases become overall attractive $(-0.5$ to $-0.7 \mathrm{eV})$, and the total energy for the $\alpha \beta$ phase is $0.22 \mathrm{eV}$ lower than that of the $\alpha \alpha$ phase, suggesting that the $\alpha \beta$ phase is more favorable under realistic conditions which explains the predominance of the $\alpha \beta$ orientation in the STM images.

Using the $\alpha \beta$ phase in Fig. 10(c), simulated STM images based on the charge densities for the HOMO and LUMO are shown in Fig. 11. The charge distribution of the HOMO

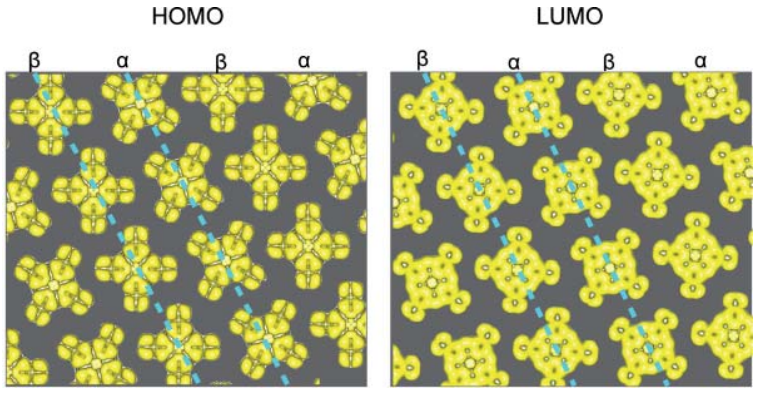

FIG. 11. Simulated STM images from the HOMO (left) and LUMO (right) states upon $\mathrm{F}_{16} \mathrm{CuPc}$ adsorption on graphene $[(3,4) \times(8,6)]$ (corresponding to applied positive and negative bias on the tip, respectively). The blue dashed lines indicate the orientations of molecular rows.

and LUMO has four-fold symmetry and a hole in the center. For the HOMO, each lobe is separated by two perpendicular planes, and the charge distribution in F-substituted phenyl groups is relatively localized. In the LUMO, the wavefunctions are more delocalized and evenly distributed over the entire Pc molecule. Furthermore, the central part of the HOMO is darker than that of the LUMO image, which is in excellent agreement as the STM images. ${ }^{30}$ The molecular arrangements and the overall features of the simulated STM image of LUMO in Fig. 11 are very close to that measured in experiment, see Fig. 5.

By increasing the $\mathrm{F}_{16} \mathrm{CuPc}$ coverage until the graphene surface is fully covered by the Pc molecules, a Pc bilayer structure begins to form with $\alpha \beta$ stripes, similar to that in the bottom layer. The interaction between stacking $\mathrm{F}_{16} \mathrm{CuPc}$ molecules is much weaker than the Pc-graphene interaction.

\section{CONCLUSIONS}

We have studied the atomic and electronic structure of $\mathrm{CuPc}$ and $\mathrm{F}_{16} \mathrm{CuPc}$ overlayers on epitaxial graphene based on STM and first-principles calculations. Both CuPc and $\mathrm{F}_{16} \mathrm{CuPc}$ molecules prefer to lie flat and physisorb with the central $\mathrm{Cu}$ atom situated on top of a $\mathrm{C}$ atom of EG. For $\mathrm{F}_{16} \mathrm{CuPc}$ adsorption on $\mathrm{EG}$, the molecules form incommensurate crystalline islands comprising alternating $\alpha$ and $\beta$-stripes. In addition, the small energy difference between these arrangements of $0.1 \mathrm{eV}$ indicates the stability of both orientations which coexist on EG. Furthermore, the order is maintained by the molecule-substrate and intermolecular van der Waals interactions and is associated with a small amount of charge transfer from EG to the $\mathrm{F}_{16} \mathrm{CuPc}$ molecule. More importantly, the negatively charged system leads to enhanced charge accumulation on Pc molecules relative to the neutral system. This voltage- and thickness-dependence of charge transfer suggests a simple way to controllably tune the amount of charge transfer between organic overlayers and graphene without resorting to complex chemical doping.

Because of the higher DOS at the Fermi energy and different amount of charge transfer, Pc molecules prefer to absorb on monolayer EG, when the monolayer and bilayer EG coexist. This paves a possible way for interfacial structure engineering. The calculated charge density of 
$\mathrm{F}_{16} \mathrm{CuPc} /$ graphene matches very well with the STM images observed in experiment.

For $\mathrm{CuPc}$ adsorption on EG, the molecules are arranged in a more compact square lattice due to their smaller size and weaker intermolecular interactions. The binding energy is $0.7 \mathrm{eV}$ smaller than that of $\mathrm{F}_{16} \mathrm{CuPc}$ on EG.

Upon Pc molecular adsorption on EG, the modification of electronic structure generates a localized molecular level at $0.4 \mathrm{eV}$ above the Dirac point in graphene, and electron redistribution below the HOMO for both cases. However, the LUMO of $\mathrm{F}_{16} \mathrm{CuPc}$ is closer to the Fermi level than that of $\mathrm{CuPc}$, indicating that the latter is more difficult to accept electrons. Furthermore, $\mathrm{F}_{16} \mathrm{CuPc}$ shows a much stronger electron polarization effect at the Pc-graphene interface than $\mathrm{CuPc}$, which also suggests the electron-acceptor character of $\mathrm{F}_{16} \mathrm{CuPc}$, and electron-donor behavior of $\mathrm{CuPc}$. The level alignments agree with experimental measurements. ${ }^{55}$

Our research elucidates the nature of the interaction between $\mathrm{F}_{16} \mathrm{CuPc}$ or $\mathrm{CuPc}$ molecules and graphene and the charge transfer mechanism at the interface. The results are useful in understanding the electronic structure of graphene tuned by physisorbed molecules and shed light on structural and electronic properties of graphene-based nanoelectronic devices.

${ }^{1}$ W. Ma, C. Yang, X. Gong, K. Lee, and A. J. Heeger, Adv. Funct. Mater. 15, 1617 (2005).

${ }^{2}$ J. Y. Kim, K. Lee, N. E. Coates, D. Moses, T. Q. Nguyen, M. Dante, and A. J. Heeger, Science 317, 222 (2007).

${ }^{3}$ J. G. Xue, S. Uchida, B. P. Rand, and S. R. Forrest, Appl. Phys. Lett. 85, 5757 (2004)

${ }^{4}$ M. A. Green, K. Emery, Y. Hishikawa, and W. Warta, Prog. Photovoltaics 17, 85 (2009).

${ }^{5}$ S. W. Cho, L. F. J. Piper, A. DeMasi, A. R. H. Preston, K. E. Smith, K. V. Chauhan, P. Sullivan, R. A. Hatton, and T. S. Jones, J. Phys. Chem. C 114, 1928 (2010)

${ }^{6}$ S. H. Park, J. G. Jeong, H.-J. Kim, S.-H. Park, M.-H. Cho, S. W. Cho, Y. Yi, M. Y. Heo, and H. Sohn, Appl. Phys. Lett. 96, 013302 (2010).

${ }^{7}$ N. R. Armstrong, W. Wang, D. M. Alloway, D. Placencia, E. Ratcliff, and M. Brumbach, Macromol. Rapid Commun. 30, 717 (2009).

${ }^{8}$ F. Yang, M. Shtein, and S. R. Forrest, Nature Mater. 4, 37 (2005).

${ }^{9}$ S. Pfuetzner, J. Meiss, A. Petrich, M. Riede, and K. Leo, Appl. Phys. Lett. 94, 253303 (2009).

${ }^{10}$ S. H. Park, A. Roy, S. Beaupre, S. Cho, N. Coates, J. S. Moon, D. Moses, M. Leclerc, K. Lee, and A. J. Heeger, Nature Photonics 3, 297 (2009).

${ }^{11}$ R. Schueppel, R. Timmreck, N. ALllinger, T. Mueller, M. Furno, C. Uhrich, K. Leo, and M. Riede, J. Appl. Phys. 107, 044503 (2010).

${ }^{12}$ H. Huang, W. Chen, and A. T. S. Wee, J. Phys. Chem. C 112, 14913 (2008).

${ }^{13}$ J. T. Sun, Y. H. Lu, W. Chen, Y. P. Feng, and A. T. S. Wee, Phys. Rev. B 81, 155403 (2010).

${ }^{14}$ S.-H. Lee, D.-H. Kim, J.-H. Kim, T.-H. Shim, and J.-G. Park, Synth. Met. 159, 1705 (2009).

${ }^{15}$ K. M. Lau, J. X. Tang, H. Y. Sun, C. S. Lee, S. T. Lee, and D. H. Yan, Appl. Phys. Lett. 88, 173513 (2006).

${ }^{16}$ A. Ruocco, F. Evangelista, R. Gotter, A. Attili, and G. Stefani, J. Phys. Chem. C 112, 2016 (2008).

${ }^{17}$ L. Yan, N. J. Watkins, S. Zorba, Y. L. Gao, and C. W. Tang, Appl. Phys. Lett. 81, 2752 (2002).

${ }^{18}$ A. S. Komolov and P. J. Moller, Synth. Met. 138, 119 (2003).
${ }^{19}$ S. Kera, Y. Yabuuchi, H. Yamane, H. Setoyama, K. K. Okudaira, A. Kahn, and N. Ueno, Phys. Rev. B 70, 085304 (2004).

${ }^{20}$ Z. N. Bao, A. J. Lovinger, and J. Brown, J. Am. Chem. Soc. 120, 207 (1998).

${ }^{21}$ Y. J. Zhang, H. L. Dong, Q. X. Tang, S. Ferdous, F. Liu, S. C. B. Mannsfeld, W. P. Hu, and A. L. Briseno, J. Am. Chem. Soc. 132, 11580 (2010).

${ }^{22}$ K. S. Novoselov, A. K. Geim, S. V. Morozov, D. Jiang, Y. Zhang, S. V. Dubonons, I. V. Grigorieva, and A. A. Firsove, Science 306, 666 (2004).

${ }^{23}$ A. K. Geim and K. S. Novoselov, Nature Mater. 6, 183 (2007).

${ }^{24}$ A. H. C. Neto, F. Guinea, N. M. R. Peres, K. S. Novoselov, and A. K. Geim, Rev. Mod. Phys. 81, 109 (2009).

${ }^{25}$ L. G. De Arco, Y. Zhang, C. W. Schlenker, K. Ryu, M. E. Thompson, and C. W. Zhou, ACS Nano 4, 2865 (2010).

${ }^{26}$ Y. Wakayama, J. Phys. Chem. C 111, 2675 (2007).

${ }^{27}$ E. Barrena, D. G. de Oteyza, H. Dosch, and Y. Wakayama, ChemPhysChem 8, 1915 (2007).

${ }^{28}$ A. Gerlach, F. Schreiber, S. Sellner, H. Dosch, I. A. Vartanyants, B. C. C. Cowie, T.-L. Lee, and J. Zegenhagen, Phys. Rev. B 71, 205425 (2005).

${ }^{29}$ D. G. de Oteyza, I. Silanes, M. Ruiz-Oses, E. Barrena, B. P. Doyle, A. Arnau, H. Dosch, Y. Wakayama, and J. E. Ortega, Adv. Funct. Mater. 19, 259 (2009).

${ }^{30}$ Y. L. Huang, W. Chen, S. Chen, and A. T. S. Wee, Appl. Phys. A 95, 107 (2009).

${ }^{31}$ Y. L. Huang, H. Li, J. Ma, H. Huang, W. Chen, S. Chen, and A. T. S. Wee, Langmuir 26, 3329 (2010).

${ }^{32}$ D. G. de Oteyza, E. Barrena, J. O. Osso, H. Dosch, S. Meyer, and J. Pflaum, Appl. Phys. Lett. 87, 183504 (2005).

${ }^{33}$ J. O. Osso, F. Schreiber, M. I. Alonso, M. Garriga, E. Barrena, and H. Dosch, Org. Electron. 5, 135, (2004).

${ }^{34}$ Y. L. Wang, J. Ren, C. L. Song, Y. P. Jiang, L. L. Wang, K. He, X. Chen, J. F. Jia, S. Meng, E. Kaxiras, Q. K. Xue, and X. C. Ma, Phys. Rev. B 82, 245420 (2010).

${ }^{35}$ J. M. Soler, E. Artacho, J. D. Gale, A. Garcia, J. Junquera, P. Ordejon, and D. Sanchez-Portal, J. Phys: Condens. Matter 14, 2745 (2002).

${ }^{36}$ G. Kresse and J. Furthmuller, Phys. Rev. B 54, 11169 (1996).

${ }^{37}$ S. D. Wang, X. Dong, C. S. Lee, and S. T. Lee, J. Phys. Chem. B 108, 1529 (2004).

${ }^{38}$ N. Troullier and J. L. Martins, Phys. Rev. B 43, 1993 (1991).

${ }^{39}$ D. M. Ceperley and B. J. Alder, J. Phys. Rev. Lett. 45, 566 (1980).

${ }^{40}$ M. Dion, H. Rydberg, E. Schroder, D. C. Langreth, and B. I. Lundqvist, Phys. Rev. Lett. 92, 246401 (2004).

${ }^{41}$ G. Roman-Perez and J. M. Soler, Phys. Rev. Lett. 103, 096102 (2009).

${ }^{42}$ D. C. Langreth, B. I. Lundqvist, S. D. Chakarova-Kack, V. R. Cooper, M. Dion, P. Hyldgaard, A. Kelkkanen, J. Kleis, L. Kong, S. Li, P. G. Moses, E. Murray, A. Puzder, H. Rydberg, E. Schroder, and T. Thonhauser, J. Phys.: Condens. Matter 21, 084203 (2009).

${ }^{43}$ A. Tsolakidis, D. Sanchez-Portal, and R. M. Martin. Phys. Rev. B 66, 235416 (2002).

${ }^{44}$ J. Heyd, G. E Scuseria, and M. Ernzerhof, J. Chem. Phys. 118, 8207 (2003).

${ }^{45}$ J. Heyd, G. E Scuseria, and M. Ernzerhof, J. Chem. Phys. 124, 219906 (2006).

${ }^{46}$ P. E. Blöchl, Phys. Rev. B 50, 17953 (1994).

${ }^{47}$ N. Marom, O. Hod, G. E. Scuseria, and L. Kronik, J. Chem. Phys. 128, 164107 (2008).

${ }^{48}$ C. J. Brown, J. Chem. Soc. A, 2488 (1968).

${ }^{49}$ L. Edwards and M. Gouterman, J. Mol. Spectrosc. 33, 292 (1970).

${ }^{50}$ E. Kol'tsov, T. Basova, P. Semyannikov, and I. Igumenov, Mate. Chem. Phys. 86, 222 (2004)

${ }^{51}$ V. W. Brar, Y. B. Zhang, Y. Yajon, T. Ohta, J. L. McChesney, A. Bostwick, E. Rotenberg, K. Horn, and M. F. Crommie, Appl. Phys. Lett. 91, 122102 (2007).

${ }^{52}$ Y. B. Zhang, V. W. Brar, F. Wang, C. Girit, Y. Yayon, M. Panlasigui, A. Zettl, and M. F. Crommie, Nat. Phys. 4, 627 (2008).

${ }^{53}$ S. Grimme, Angew. Chem. Int. Ed. 47, 3430 (2008)

${ }^{54}$ S. E. Wheeler and K. N. Houk, J. Phys. Chem. A 114, 8658 (2010).

${ }^{55}$ W. Chen, H. Huang, S. Chen, Y. L. Huang, X. Y. Gao, and A. T. S. Wee, Chem. Mater. 20, 7017 (2008). 